\title{
Generation of additive colors with near unity amplitude using a multilayer tandem Fabry-Perot cavity
}

\author{
Ali Cahit Kosger, ${ }^{1,2, *}$ Amir Ghobadi, ${ }^{1,3}$ (1) Alireza Rahimi Rashed, ${ }^{4}$ (i) \\ Humeyra Caglayan, ${ }^{4}$ (1) and Ekmel Ozbay ${ }^{1,2,3,5}$ \\ ${ }^{1}$ NANOTAM-Nanotechnology Research Center, Bilkent University, 06800 Ankara, Turkey \\ ${ }^{2}$ UNAM - National Nanotechnology Research Center, Institute of Materials Science and Nanotechnology, Bilkent University, 06800 Ankara, \\ Turkey \\ ${ }^{3}$ Department of Electrical and Electronics Engineering, Bilkent University, 06800 Ankara, Turkey \\ ${ }^{4}$ Faculty of Engineering and Natural Sciences, Photonics, Tampere University, 33720 Tampere, Finland \\ ${ }^{5}$ e-mail: Ozbay@bilkent.edu.tr \\ ${ }^{*}$ Corresponding author: kosger@bilkent.edu.tr
}

Received 7 May 2021; revised 5 June 2021; accepted 16 June 2021; posted 17 June 2021 (Doc. ID 430985$) ;$ published 15 July 2021

In this paper, the generation of additive red-green-blue (RGB) colors in reflectance mode with near unity amplitude is demonstrated. For this purpose, a multilayer structure made of metal-insulator-metal-semiconductor-insulator stacks is designed to achieve $>0.9$ reflection peaks with full-width-at-half-maximum values $<0.3 \lambda_{\text {peak }}$. The proposed design also shows near zero reflection in off-resonance spectral ranges, and this, in turn, leads to high color purity. The optimized designs are fabricated, and simulation and theoretical results are verified with characterization findings. This work demonstrates the potential of multilayer tandem cavity designs in the realization of lithography-free large-scale compatible functional optical coatings. () 2021 Optical Society of America

https://doi.org/10.1364/OL.430985

Up to now, various design architectures have been utilized to fabricate color filters in reflection and transmission modes. Designs operating based on surface plasmon resonance [1,2], guided-mode resonance $[3,4]$, and photonic crystals $[5,6]$ as well as other innovative ideas $[7,8]$ are some of these approaches. However, in all of these designs, the color generation is accomplished via spectrally selective light-matter interaction in sub-wavelength metal-dielectric nanounits. The realization of these nanounits, however, requires complex fabrication routes, such as electron-beam lithography (EBL) and nanoimprint lithography (NIL), which are large-scale incompatible approaches. Therefore, lithography-free design structures have attracted much attention in recent years [9].

Among all resonators, metal-insulator (MI) pair based Fabry-Perot (FP) resonators are the most commonly employed designs for large-scale color generation [10-14]. These multilayer designs provide strong interference in spectrally selective regions. Generally, these planar designs produce additive redgreen-blue (RGB) colors in transmission mode, and subtractive cyan-magenta-yellow (CMY) colors in reflection mode. Unlike reflection mode designs that can reach near unity amplitude, transmissive RGB colors have efficiencies lower than $\sim 0.7$, which is due to the existence of multiple lossy layers in their propagation paths. To tackle this deficiency, proposing a planar lithography-free architecture to generate RGB colors in the reflection mode is a possible solution. The use of lossy metals such as bismuth [15], nickel [16,17], and platinum [18] as the top layer in a MI-metal (MIM) design has been proposed to achieve this goal. However, the wide full-width-at-halfmaximum (FWHM) of these designs reduces the color purity. Other innovative architectures have also been explored to generate RGB colors in reflection mode. The use of metal-dielectric nanodesigns [19-21], multilayer MI pair based architectures such as MIMIM [22], and other innovative architectures $[23,24]$ are some examples of these ideas. However, most of these designs have low efficiencies and/or their color purity is poor.

In this Letter, the generation of RGB colors is numerically and experimentally demonstrated using a designed metaldielectric medium. For this, first, a modeling approach based on the transfer matrix method (TMM) is developed to compare the performance of common MIM cavities with those of MIMI ones. Later, using numerical simulations, the optimal geometries are extracted. Gaining knowledge from these results, a hybrid structure made of MI-metal-semiconductor-insulator (MIMSI) stacks is optimized to create RGB colors with high purity and near unity amplitude. Finally, the optimal geometries are utilized to fabricate RGB color filters with an amplitude $>0.9$ reflection peak and FWHM $<0.3 \lambda_{\text {peak }}$. The findings of this study show that a proper connection of cavities in a tandem scheme can offer diverse functionalities that cannot be realized with single FP resonators.

To begin with, the reflection characteristics of two common FP resonators of MIM and MIMI are scrutinized using a modeling approach based on TMM [Figs. 1(a)-1(f)]. The details of this modeling have been explained in our previous 
studies [24]. In this modeling, the bottom layer is fixed as an optically thick aluminum mirror, the spacer thickness $\left(D_{I}\right)$ is set as $60 \mathrm{~nm} \mathrm{Al} \mathrm{O}_{3}$ layer, and the reflection contour plots for different top metal thicknesses $\left(D_{M}\right)$ of $5 \mathrm{~nm}$ and $15 \mathrm{~nm}$ are extracted. These contour plots are obtained for five different wavelength values of $450 \mathrm{~nm}, 500 \mathrm{~nm}, 550 \mathrm{~nm}, 600 \mathrm{~nm}$, and $650 \mathrm{~nm}$. In these $2 \mathrm{D}$ plots, the centric blue semi-circles show the real and imaginary permittivity values that are required to have $<0.1$ reflection (absorption $>0.9$ ). Comparing these two designs, it can be understood that the tolerable region for light perfect absorption is wider for the MIMI design (compared to that of MIM). Moreover, thicker $D_{M}$ values lead to wider perfect absorption circles. In addition, these circles are placed in the vicinity of the center, and a lossy metal with real and imaginary parts close to zero provides better performance. Therefore, from these modeling findings, in an MIMI absorber made of a lossy metal, constructive interference at a specific wavelength will lead to near unity reflection, while the other parts of the spectrum will be perfectly absorbed. In this way, an RGB color filter, in the reflective mode, with high efficiency and color purity can be generated.

To verify these modeling outputs, numerical simulations using the Lumerical finite-difference-time-domain (FDTD) software package are performed [25]. In the simulations, the bottom layer is chosen as a $150 \mathrm{~nm}$ thick $\mathrm{Al}$ layer. The insulator layers are set as $\mathrm{Al}_{2} \mathrm{O}_{3}$ with identical thicknesses of $D_{I}$. Finally, the top metal layer is $\mathrm{Ni}$ with thickness of $D_{M}$. All the permittivity data are extracted from the Palik model [26]. In this design, (a)
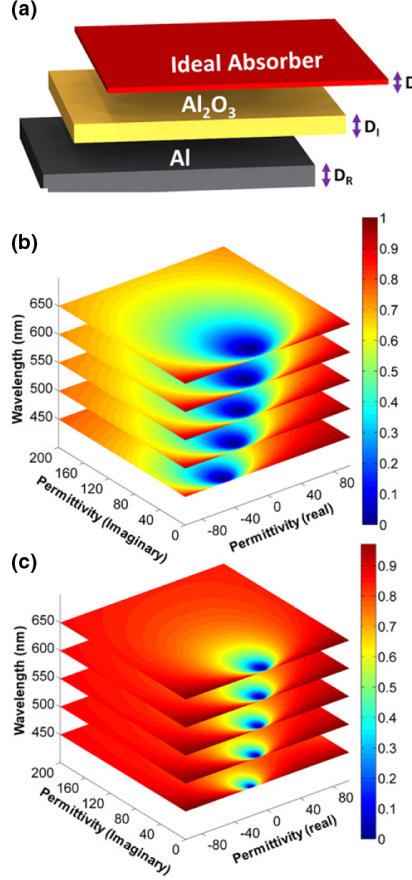

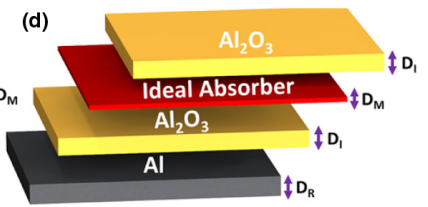

(e)

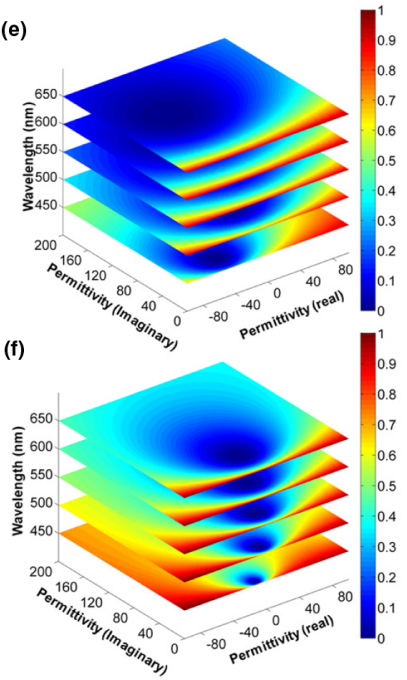

Fig. 1. Schematic representation of (a) MIM cavity design. Reflection contour plots of an MIM cavity at different real and imaginary permittivity values for $D_{M}$ of (b) $5 \mathrm{~nm}$ and (c) $15 \mathrm{~nm}$. Schematic representation of (d) MIMI cavity design. Reflection contour plots of an MIM cavity at different real and imaginary permittivity values for $D_{M}$ of (e) $5 \mathrm{~nm}$ and (f) $15 \mathrm{~nm}$. In all of the modeling results, $\left(D_{I}\right)$ is set as the $60 \mathrm{~nm} \mathrm{Al}_{2} \mathrm{O}_{3}$ layer, and the bottom layer is an optically thick $\mathrm{Al}$ layer. The color bar shows the reflection value of the cavity. two main geometries of $D_{I}$ and $D_{M}$ are responsible for the optical behavior of the cavities. First, the insulator layer thickness is fixed, and the metal layer is swept to find the optimal value for light perfect absorption. Later, the metal layer thickness is fixed at the optimum value, and the spectral shift of this resonance peak in different insulator layer thicknesses is studied. As shown in Fig. 2(a), for MIM design, $D_{I}$ is fixed at $150 \mathrm{~nm}$ and $D_{M}$ is swept from $3 \mathrm{~nm}$ to $18 \mathrm{~nm}$, with a step of $5 \mathrm{~nm}$. As this panel implies, the reflection peak amplitude is almost constant for all $D_{M} s$, and thicker layers lead to narrower FWHM. However, at the expense of this narrowing, the non-resonant response (reflection ripples in the non-resonant spectral range) becomes larger, and this, in turn, reduces the color purity of the filter. Therefore, in an appropriate metal thickness layer, we can provide a right trade-off between these two factors. This trade-off is satisfied in $D_{M}=6 \mathrm{~nm}$ for the MIM cavity absorber. Setting this as the top layer, next, $D_{I}$ is swept from $100 \mathrm{~nm}$ to $200 \mathrm{~nm}$ (with $25 \mathrm{~nm}$ step). In Fig. 2(b), the reflection peak is monolithically shifted from blue to red wavelengths, without any loss in its amplitude. However, blue filters (lower wavelength ranges) suffer from non-resonant absorptions (in the longer visible range), and this leads to poorer color pureness. A similar analysis is performed on an MIMI cavity absorber, as shown in Figs. 2(c)-2(d). For MIMI color filters, the optimal $D_{M}$ is $9 \mathrm{~nm}$. Compared with MIM cases, MIMI designs have smaller FWHM, but similar to them, they also have non-resonant absorption ripples, and, therefore, they have low color vividness.

Considering all the above findings, the MIMI configuration based color filters have better performance in RGB color generation compared to that of MIM ones. However, both designs have poor color purity, and using a conventional single cavity FP resonator, this property cannot be achieved. Therefore, the simultaneous realization of color purity and efficiency needs a tandem scheme FP design. For this purpose, a multilayer design based on MIMSI stacks is developed, as seen in Fig. 2(e). Although, some recent studies [27,28] have demonstrated that the optimization of top insulator layer thickness (for each color filter) can improve color purity, to have a better qualitative comparison between different configurations, we use identical $D_{I}$ thicknesses for both insulator layers. The semiconductor layer is Ge with a thickness of $5 \mathrm{~nm}$. All other geometries are set at the optimal values of the MIMI structure. The reflection spectra of MIMI and MIMSI designs are compared in Fig. 2(f). As this figure depicts, the addition of an ultrathin Ge layer not only lowers the FWHM of the reflection peak, but also effectively mitigates the non-resonant absorption ripples. To understand the function of this Ge layer on the overall optical performance of the MIMSI FP cavity, the absorbed power across the MIMI and MIMSI cavities is plotted in Figs. 2(g)-2(h). It should be mentioned that in these contour plots, the profile is zoomed on the active middle layer, and these data belong to the case of a red filter. In these panels, the Ge layer harnesses most of the incoming power in the shorter $\lambda$ values, and $\mathrm{Ni}$ is the active material in the longer ranges, while both of these layers are transparent in the peak resonance position. This can be seen in the linear plots as well [Fig. 2(f)], where the sub-peaks (nonresonant modes) in the reflection of MIMI are suppressed by MIMSI design. Therefore, using this designed FP resonator, it is possible to achieve large-scale compatible and high efficiency RGB vivid colors in the reflection mode color filters. It should be mentioned that the order in placement of the $\mathrm{Ni}-\mathrm{Ge}$ bilayer is 

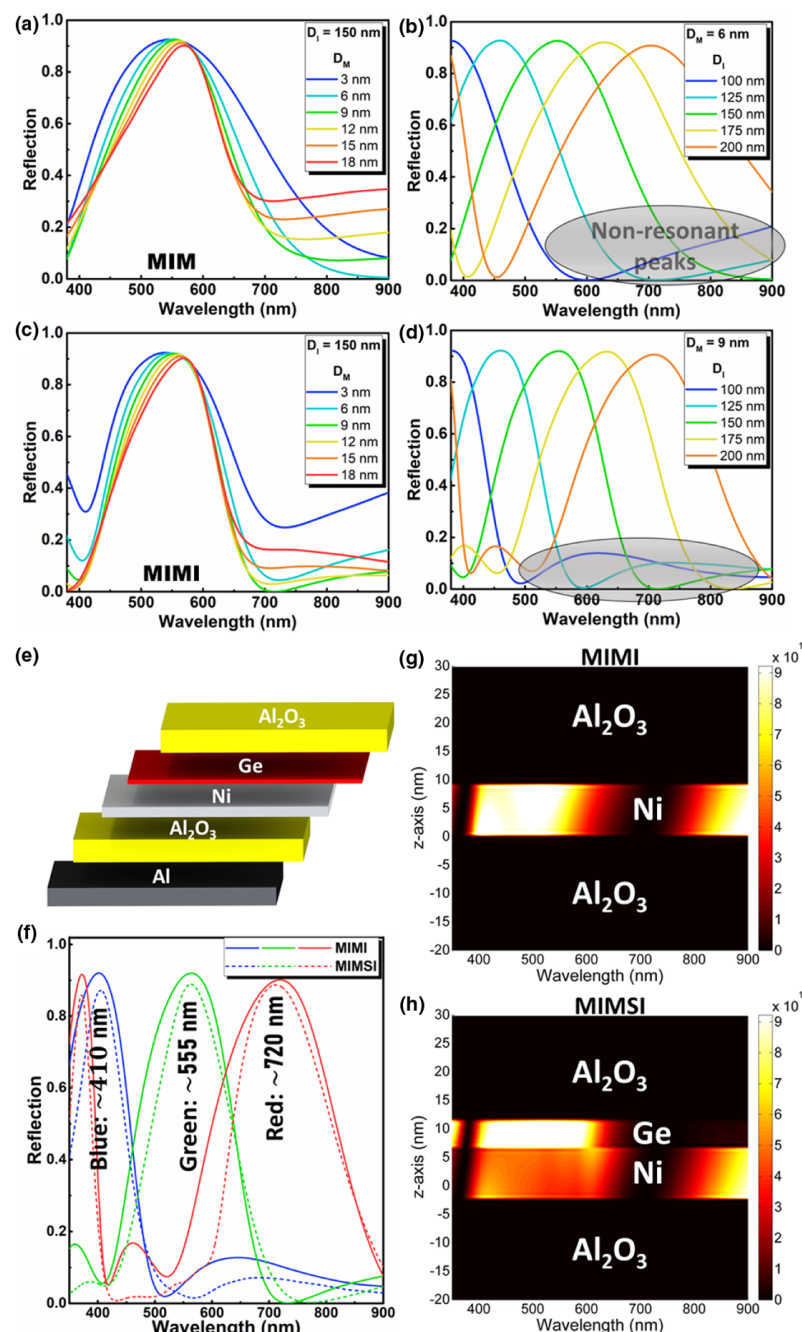

\section{(h)}

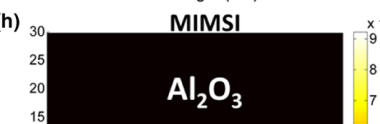

高窟
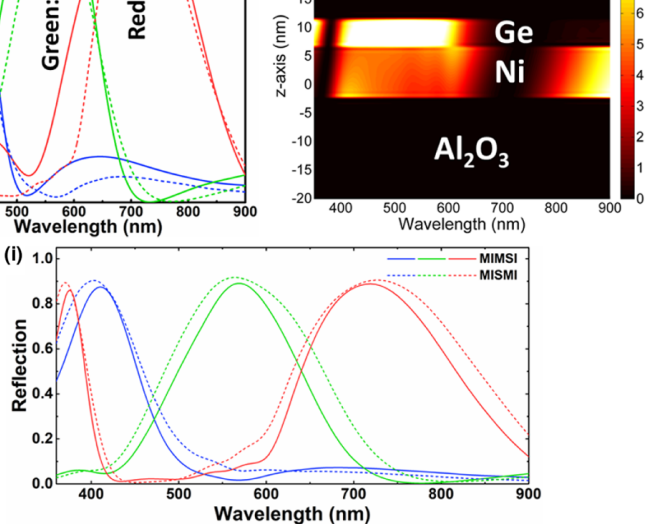

Fig. 2. Reflection spectra of MIM cavity design for different (a) $D_{M}$ and (b) $D_{I}$ values. Reflection spectra of MIMI design for different (c) $D_{M}$ and (d) $D_{I}$ values. The highlighted regions show the nonresonant ripples. (e) Schematic representation of MIMSI cavity design; (f) reflection spectra of RGB color filters for MIMI and MIMSI structures; absorption profile across the cavity structure for (g) MIMI and (h) MIMSI color filters. The color bar shows the absorbed power value. (i) Comparison between MIMSI and MISMI configurations.

also optimized. As shown in Fig. 2(i), both configurations have similar responses; however, the MIMSI design has narrower spectral peaks. Placing Ge on top of the metal layer (forming a metal-semiconductor nanocavity) provides broadband stronger interference, and in this way, the absorption performance of the design is improved, and it shows itself as a narrower reflection peak.

In the next step, the simulation data have to be verified by experimental data. For this, a set of MIMSI samples with optimized $D_{M}$ values of $9 \mathrm{~nm}$ and different $D_{I}$ thicknesses (from $110 \mathrm{~nm}$ to $200 \mathrm{~nm}$ by a step of $15 \mathrm{~nm}$ ) is fabricated using cleanroom facilities. To have a better qualitative comparison with a common FP color filter, a similar set is also fabricated for MIM architecture. For the fabrication, an $\mathrm{Si}$ wafer is diced into $1 \mathrm{~cm} \times 1 \mathrm{~cm}$ pieces, and a standard cleaning process is applied on them. The metallic (both $\mathrm{Al}$ and $\mathrm{Ni}$ ) and Ge layers are coated at the desired thicknesses, using the thermal evaporation technique. The deposition rate is set at around $0.8-1.2 \mathrm{~A} / \mathrm{s}$ for all layers and the chamber pressure is kept below 5e-6 Torr throughout the deposition process. It should be mentioned that although the Ge layer is quite thin, the deposition rate is kept slow enough to have a conformal coating. Moreover, our MIMSI design can tolerate variations in Ge thickness. For $\mathrm{Al}_{2} \mathrm{O}_{3}$ coating, atomic layer deposition (Cambridge Nanotech Savannah S100) is used at $250^{\circ} \mathrm{C}$ using $\mathrm{Al}\left(\mathrm{CH}_{3}\right)_{3}$ and water precursors as aluminum and oxygen sources. The pulse and purge times are chosen to be $0.015 \mathrm{~s}$ and $10 \mathrm{~s}$. Optical images of fabricated MIM and MIMSI samples are shown in Fig. 3(a). From their visual appearance, it can be deduced that MIMSI cavities have better color vividness compared to MIM ones. To verify the formation of different layers, scanning electron microscopy (SEM) imaging is utilized. Cross-sectional SEM images of the MIM and MIMSI samples, shown in Figs. 3(b)

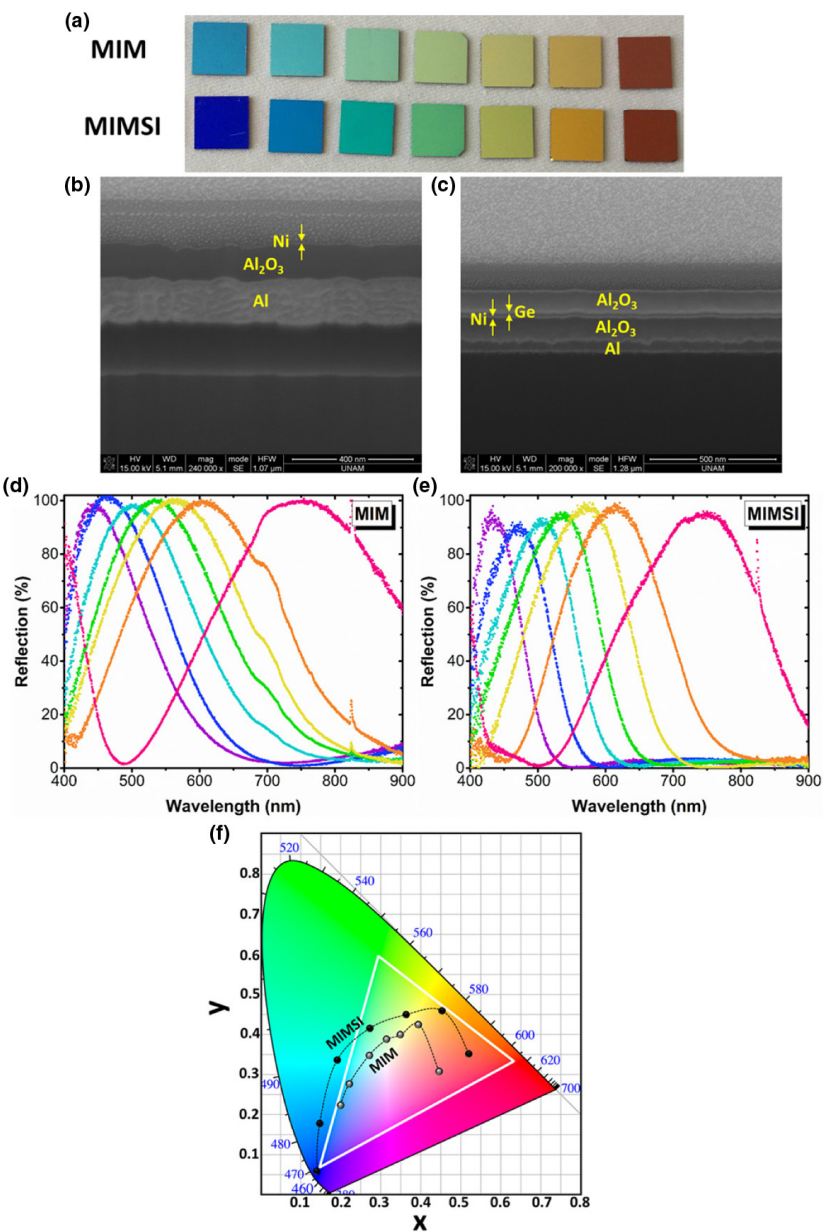

Fig. 3. (a) Optical image of MIM and MIMSI samples with different spacer thicknesses to generate RGB colors. Cross-sectional SEM image of (b) MIM and (c) MIMSI cavity designs. Measured reflection spectra of different (d) MIM and (e) MIMSI samples. (f) Color coordinates of MIM and MIMSI color filters in the 1931 CIE color diagram. 

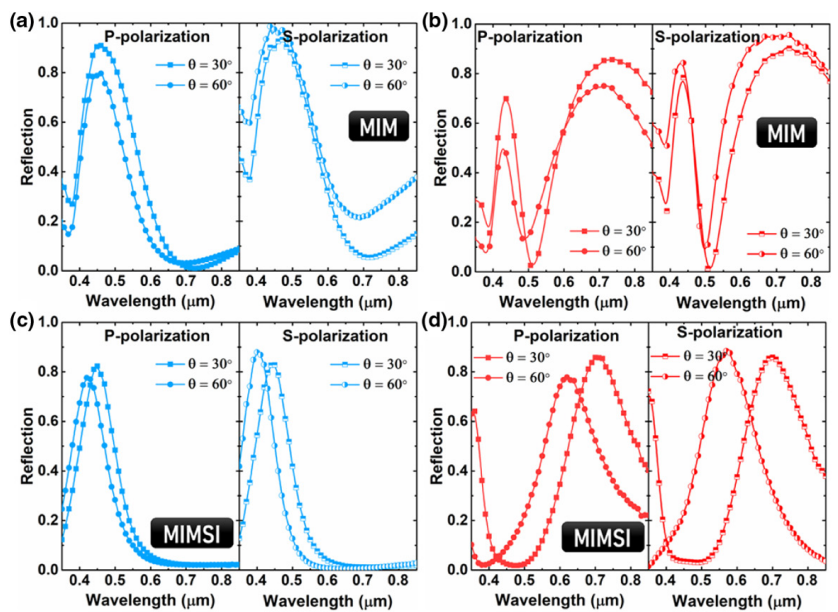

Fig. 4. Angular response of the MIM filter for two different colors of (a) blue and (b) red. Angular response of the MIMSI structure for two different colors of (c) blue and (d) red. The measurements are carried out at both $p$ and $s$ polarizations and two different incident angles of $30^{\circ}$ and $60^{\circ}$.

and 3(c), confirm successful growth of all layers. After the initial structural analysis, optical characterizations are utilized to probe the reflection response of each sample. As can be seen in Fig. 3(d), the MIM color filter has reflection peaks above 0.95. This peak value is gradually reduced, but it still stays above 0.9 for MIMSI cavity designs [Fig. 3(e)]. Moreover, the color coordinates of the RGB color filters generated by MIM and MIMSI stacks are presented in the 1931 CIE color diagram to compare their color purity [Fig. 3(f)]. As this panel implies, the MIMSI design has better matching with a standard RGB (sRGB) triangle. However, in a one-by-one comparison, it can be found that MIMSI designs have considerably lower FWHMs and quite small non-resonance reflection colors. For instance, in the case of the blue color filter (for $D_{I}=110 \mathrm{~nm}$ ), the FWHMs for MIM and MIMSI samples are $131 \mathrm{~nm}\left(0.3 \lambda_{\text {peak }}\right)$ and $77 \mathrm{~nm}$ $\left(0.17 \lambda_{\text {peak }}\right)$, respectively. These values for green $\left(D_{I}=155 \mathrm{~nm}\right)$ are found to be $231 \mathrm{~nm}\left(0.43 \lambda_{\text {peak }}\right)$ and $141 \mathrm{~nm}\left(0.26 \lambda_{\text {peak }}\right)$, and red $\left(D_{I}=200 \mathrm{~nm}\right)$ are $323 \mathrm{~nm}\left(0.43 \lambda_{\text {peak }}\right)$ and $228 \mathrm{~nm}$ $\left(0.3 \lambda_{\text {peak }}\right)$ for MIM and MIMSI samples, respectively. Another factor that should be considered in the performance analysis of these FP filters is their angular response. For this, ellipsometry measurements are carried out in two incidence angles of $30^{\circ}$ and $60^{\circ}$ at $s$ and $p$ polarizations for both MIM and MIMSI designs with blue and red color filters [Figs. 4(a)-4(d)]. As these panels imply, overall, the resonance wavelength is shifted toward the blue region in both cases. These shifts are more pronounced in the MIMSI designs due to the larger optical path of light in these samples (compared to MIM ones). However, the color purity (peak shape and non-resonant response) is significantly modified in the MIM designs, while MIMSIs have kept their peak shape and FWHM width almost intact. This is due to the fact that in an MIMSI structure, the top insulator layer acts as a broadband antireflective coating that provides phase matching conditions in a broad wavelength and angles.

Overall, this work demonstrates the effectiveness of tandemshaped FP designs in achieving optical behaviors that cannot be realized with common cavity designs. The high efficiency and color purity of these planar MIMSI designs, together with their large-scale compatibility, give them the opportunity to be scaled up for commercial applications.

Disclosures. The authors declare no conflict of interest.

Data Availability. Data underlying the results presented in this paper are not publicly available at this time but may be obtained from the authors upon reasonable request.

\section{REFERENCES}

1. D. Fleischman, L. A. Sweatlock, H. Murakami, and H. Atwater, Opt. Express 25, 27386 (2017).

2. Y. Gu, L. Zhang, J. K. W. Yang, S. P. Yeo, and C.-W. Quu, Nanoscale 7, 6409 (2015).

3. Y.-T. Yoon, H.-S. Lee, S.-S. Lee, S. H. Kim, J.-D. Park, and K.-D. Lee, Opt. Express 16, 2374 (2008).

4. M. J. Uddin, T. Khaleque, and R. Magnusson, Opt. Express 22, 12307 (2014).

5. E.-H. Cho, H.-S. Kim, B.-H. Cheong, P. Oleg, W. Xianyua, J.-S. Sohn, D.-J. Ma, H.-Y. Choi, N.-C. Park, and Y.-P. Park, Opt. Express 17, 8621 (2009).

6. H. Cho, S. Han, J. Kwon, J. Jung, H.-J. Kim, H. Kim, H. Eom, S. Hong, and S. H. Ko, Opt. Lett. 43, 3501 (2018).

7. W. Yue, S. Gao, S. S. Lee, E. S. Kim, and D. Y. Choi, Sci. Rep. 6, 29756 (2016).

8. K.-T. Lee, J.-Y. Jang, S. J. Park, C. Ji, L. J. Guo, and H. J. Park, Optica 3, 1489 (2016).

9. A. Ghobadi, H. Hajian, B. Butun, and E. Ozbay, ACS Photon. 5, 4203 (2018).

10. C. S. Park, V. R. Shrestha, S. S. Lee, and D. Y. Choi, Sci. Rep. 6, 25496 (2016).

11. Z. Li, S. Butun, and K. Aydin, J. Opt. 18, 065006 (2016).

12. Z. Li, S. Butun, and K. Aydin, ACS Photon. 2, 183 (2015).

13. K. T. Lee, S. Y. Han, and H. J. Park, Adv. Opt. Mater. 5, 1700284 (2017).

14. K. T. Lee, S. Seo, J. Y. Lee, and L. J. Guo, Appl. Phys. Lett. 104, 231112 (2014).

15. A. Ghobadi, H. Hajian, M. Gokbayrak, B. Butun, and E. Ozbay, Nanophotonics 8, 823 (2019).

16. Z. Yang, Y. Zhou, Y. Chen, Y. Wang, P. Dai, Z. Zhang, and H. Duan, Adv. Opt. Mater. 4, 1196 (2016).

17. S. Daqiqeh Rezaei, J. Ho, T. Wang, J. K. W. Yang, and S. Ramakrishna, Nano Lett. 20, 4422 (2020).

18. C. S. Park and S. S. Lee, ACS Appl. Nano Mater. 4, 4216 (2021).

19. H. S. Song, G. J. Lee, D. E. Yoo, Y. J. Kim, Y. J. Yoo, D. W. Lee, V. Siva, I. S. Kang, and Y. M. Song, Sci. Rep. 9, 3350 (2019).

20. C.-S. Park, I. Koirala, S. Gao, V. R. Shrestha, S.-S. Lee, and D.-Y. Choi, Opt. Express 27, 667 (2019).

21. M. Jiang, S. Y. Siew, J. Y. E. Chan, J. Deng, Q. Y. S. Wu, L. Jin, J. K. W. Yang, J. Teng, A. Danner, and C. W. Qiu, Mater. Today 35, 99 (2020).

22. D. H. Kim, Y. J. Yoo, J. H. Ko, Y. J. Kim, and Y. M. Song, Opt. Mater. Express 9, 3342 (2019).

23. A. Ghobadi, H. Hajian, M. C. Soydan, B. Butun, and E. Ozbay, Sci. Rep. 9, 290 (2019).

24. C. Ji, K.-T. Lee, and L. J. Guo, Opt. Lett. 44, 86 (2019).

25. Lumerical Solut. Inc. https://www.Lumerical.Com/TcadProducts/Fdtd/.

26. E. D. Palik, Handbook of Optical Constants of Solids, (Academic, 2012).

27. Z. Yang, C. Ji, Q. Cui, and L. J. Guo, Adv. Opt. Mater. 8, 200031 (2020).

28. Z. Yang, C. Ji, D. Liu, and L. J. Guo, Adv. Opt. Mater. 7, 1900739 (2019). 